\title{
Análisis y modelado de un sistema para tomografía optoacústica basado en interferometría óptica heterodina
}

\author{
Analysis and modeling of a system for optoacoustic tomography based on heterodyne optical \\ interferometry
}

\author{
Roberto M. Insabella*1, Martín G. González*† \\ *Universidad de Buenos Aires, Facultad de Ingeniería, \\ Grupo de Láser, Óptica de Materiales y Aplicaciones Electromagnéticas (GLOmAe) \\ Paseo Colón 850, C1063ACV, Buenos Aires, Argentina \\ ${ }^{\dagger}$ Consejo Nacional de Investigaciones Científicas y Técnicas, (CONICET) \\ Godoy Cruz 2290, C1425FQB, Buenos Aires, Argentina \\ 1 rinsabellaefi.uba.ar \\ Recibido: 02/11/21; Aceptado: 01/12/21
}

\begin{abstract}
In this work, the source of the artifacts introduced in the images obtained with an optoacoustic tomography system based on the software-defined optoelectronics concept are analyzed and characterized. It is shown that the measured signals are affected both by the cylindrical geometry of the optical sensor and by electrical noise. The latter has well-defined frequencies within the spectrum caused by the electronics used in the heterodyning process of the ultrasound optical detector. A way to include these effects in simulated signals is proposed and the model is tested against measurements. The results of this work will allow the use of the deep learning technique to improve the quality of the images obtained with this type of tomographic systems.
\end{abstract}

Keywords: optoacoustic; interferometer; electrical noise.

Resumen- En este trabajo se analizan y caracterizan las fuentes de los artefactos introducidos en las imágenes obtenidas con un sistema para tomografía optoacústica basado en el concepto de optoelectrónica definida por software. Se muestra que las señales medidas están afectadas tanto por la geometría cilíndrica del sensor óptico como por el ruido eléctrico. Este último posee frecuencias bien definidas dentro del espectro atribuibles a la electrónica usada en el proceso de heterodinaje del detector óptico de ultrasonido. Se propone una forma de incluir estos efectos en señales simuladas y se prueba el modelo comparándolo con mediciones. Los resultados de este trabajo permitirán el uso de la técnica de aprendizaje profundo para mejorar la calidad de las imágenes obtenidas con este tipo de sistemas tomográficos.

Palabras clave: optoacústica; interferómetro; ruido eléctrico.

\section{INTRODUCCIÓN}

El efecto optoacústico (OA) es el nombre que se le da al fenómeno por el cual la absorción de un pulso óptico genera un pulso acústico. Un pulso de luz que incide en el tejido biológico blando se esparcirá por el mismo y una parte será absorbida por moléculas presentes en la muestra biológica, conocidas como cromóforos (la hemoglobina es una de las más importantes). La energía del cromóforo excitado se convierte luego en calor. Todo esto ocurre en una escala de tiempo (nanosegundos) que es mucho más corta que la escala de tiempo requerida para que cambie la densidad del medio (microsegundos), por lo que el calentamiento es isocórico $\mathrm{y}$, por lo tanto, se genera un aumento de presión. Como el tejido es elástico, las regiones de mayor presión actuarán como fuentes de ondas acústicas [1]. Las ondas acústicas son sensibles a la velocidad del sonido y la densidad del medio y estos parámetros suelen variar con la posición. Sin embargo, en tejidos blandos, las variaciones suelen ser pequeñas $y$, como rara vez se conocen de antemano, el medio suele tratarse como acústicamente homogéneo [2].

Un enfoque muy prometedor para obtención de imágenes biológicas, basado en el efecto OA y diferente a las modalidades ya establecidas (tomografía computada de rayos $\mathrm{X}$, la resonancia magnética nuclear o la obtención de imagen por ultrasonido), es la tomografía optoacústica (TOA) [1]. La TOA consiste en la resolución de un problema inverso en donde se generan ondas acústicas por efecto OA que son medidas por sensores de ultrasonido ubicados en múltiples locaciones alrededor del objeto o muestra biológica. El objetivo es conocer la distribución espacial de la absorción óptica a partir de las mediciones captadas por los sensores, utilizando un método de reconstrucción adecuado. En un esquema de TOA típico, el espécimen o fantoma bajo estudio se encuentra rodeado de agua de forma de maximizar el acoplamiento de las ondas ultrasónicas con el sistema de detección [3].

Existen dos grandes causas que limitan el desempeño de un sistema para TOA: las características del detector ultrasónico y el ruido. La forma y el tamaño de los detectores usados en TOA tienen una fuerte influencia en el proceso de reconstrucción de la imagen. Se considera un sensor ideal a un detector puntual con ancho de banda infinito y omnidireccional [4]. En la práctica, los transductores tienen un tamaño finito, un ancho de banda limitado y poseen una determinada respuesta espacial [5]. Generalmente, el sensor tiene un tamaño que no es despreciable con respecto a las dimensiones del objeto bajo estudio. Bajo esta condición, se dice que el detector es extenso o de gran área y la señal recibida a un cierto tiempo está dada por el valor instantáneo 
de la integral del campo acústico sobre su área activa. Por lo tanto, la forma del sensor tiene una fuerte influencia en el perfil temporal de la señal OA medida [6], [7].

El ruido eléctrico suele ser la principal fuente de corrupción en las señales OA medidas y surge debido a los efectos térmicos (ruido térmico) y a la interferencia electromagnética (ruido parásito). Este último posiblemente generado por el propio sistema OA o el medio ambiente [8]. Mientras que el ruido térmico puede modelarse como ruido blanco gaussiano [9], el ruido parásito implica correlaciones espacio-temporales complejas y, por lo tanto, no puede ser capturado de manera eficiente por un modelo analítico [8]. Tanto el ruido térmico como el parásito provocan artefactos en las imágenes $\mathrm{OA}$ reconstruidas que disminuyen gravemente el contraste morfológico y espectral [10]. Mientras que un adecuado blindaje puede suprimir algunos ruidos parásitos, esta solución es específica del dispositivo y, a menudo, es incompleta [8]. Por lo tanto, se necesitan técnicas de procesamiento de señales para eliminar tanto el ruido parásito como el térmico. El filtrado en frecuencia mediante filtros pasabanda no puede separar adecuadamente el ruido térmico y parásito de las señales OA porque el contenido espectral de las señales OA y el ruido se superponen significativamente. Por esta razón, los métodos de regularización y promediado son los más comúnmente aplicados para minimizar los efectos del ruido eléctrico en la obtención de imágenes OA [11]. Si bien el promediado reduce efectivamente el ruido eléctrico de media cero, este enfoque conlleva a un mayor tiempo de adquisición que aumenta la vulnerabilidad a los artefactos de movimiento, particularmente en aplicaciones clínicas o cuando se usa un sistema portátil [10]. La regularización de las reconstrucciones basadas en modelos matriciales iterativos puede disminuir los efectos del ruido eléctrico pero es un enfoque computacionalmente intensivo y, por lo tanto, no es adecuado para aplicaciones que requieren la obtención de imágenes en tiempo real [10]. Otro método para reducir el ruido se basa en representaciones dispersas de las señales OA, típicamente basadas en Wavelets [12]. Sin embargo, estos esquemas no tienen un buen rendimiento ante ruidos parásitos.

Recientemente, los enfoques basados en redes neuronales profundas (deep learning) han logrado un rendimiento de vanguardia en tareas de eliminación de ruido en imágenes [2]. Mediante el uso de enormes conjuntos de datos, se pueden entrenar estas redes para eliminar el ruido de manera más precisa, robusta y rápida que los métodos tradicionales. En este sentido, es necesario contar con una base de datos que permita ajustar los parámetros de la red neuronal para conseguir el objetivo deseado. Conseguir la gran cantidad de datos experimentales necesarios para entrenar eficientemente estas redes es una tarea dificultosa. Un enfoque es realizar un primer entrenamiento con simulaciones y luego un ajuste fino de los parámetros usando las pocas mediciones reales (fine-tuning).

En un trabajo previo [13], se presentó la primera aplicación del concepto optoelectrónica definida por software (SDO, por sus siglas en inglés) a un sistema para TOA bidimensional (2-D) basado en interferometría óptica heterodina. El concepto SDO se refiere a sistemas optoelectrónicos
TOA-SDO

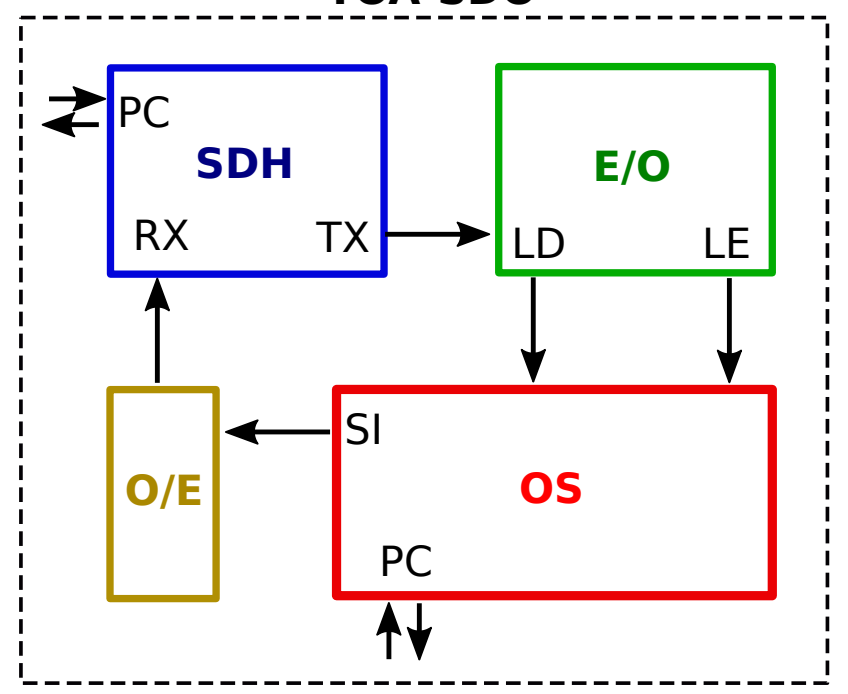

Fig. 1. Esquema del sistema TOA-SDO estudiado en este trabajo.

donde la funcionalidad asociada con el acondicionamiento y procesamiento de señales ópticas y eléctricas se implementa digitalmente y se controla mediante software. Este paradigma aprovecha la flexibilidad de las plataformas de hardware definidas por software para desarrollar sistemas de instrumentación adaptables. Las principales limitaciones en este sistema TOA basado en SDO están relacionadas con la forma del sensor ultrasónico utilizado para detectar las señales OA y en la baja relación señal a ruido (SNR) debido a la presencia de ruidos eléctricos en las mediciones. En este sentido, en este trabajo se presenta un análisis y modelado de estas dos causas que producen una baja calidad en las imágenes OA obtenidas. Asimismo, el modelo desarrollado se prueba comparándolo con imágenes obtenidas a partir de mediciones realizadas con el sistema para TOA bajo estudio.

\section{MÉtodos}

\section{A. Sistema TOA-SDO}

En la Fig. 1 se muestra que el esquema del sistema TOA está compuesto por cuatro elementos fundamentales: el sistema óptico (OS), el bloque electro-óptico (E/O), el bloque optoelectrónico $(\mathrm{O} / \mathrm{E})$ y el hardware definido por software (SDH). Este último es una plataforma comercial de radio definida por software (SDR, por sus siglas en inglés) [14] que se encarga de la modulación de la señales ópticas generadas en el bloque E/O (TX) y del procesamiento de las señales eléctricas recibidas desde el bloque O/E (RX). El puerto PC denota la comunicación de la SDR con la computadora, encargada de la configuración de la SDR y del procesamiento de las mediciones para obtener la imagen OA. El bloque E/O está compuesto por dos láseres que son utilizados para la excitación de la muestra (láser Nd:YAG pulsado doblado en frecuencia, LE) y para el sistema óptico interferómetrico encargado de detectar las señales OA (láser HeNe rojo polarizado, LD). El haz de este último pasa por un modulador acusto-óptico cuya frecuencia es controlada por el bloque SDH. El bloque OS está integrado por un interferómetro óptico en una configuración Mach-Zehnder y por un recipiente con agua (volumen de control) que 


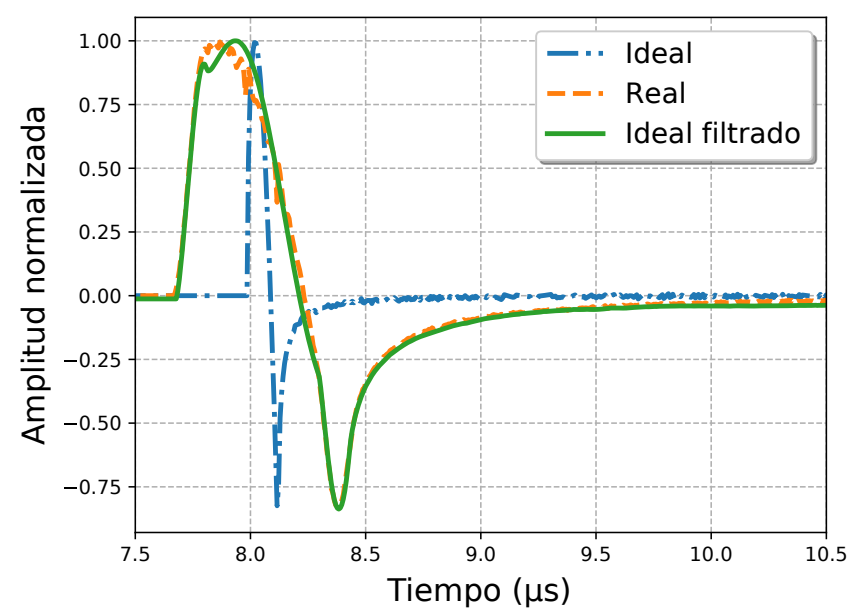

Fig. 2. Comparación entre sensor lineal ideal y real. También se muestra el resultado de simular este efecto con un filtro gaussiano 1-D truncado.

contiene el fantoma bajo estudio. Para medir la señal OA en distintas ubicaciones, el fantoma se encuentra adherido sobre una plataforma giratoria controlada por computadora (PC). Se ha demostrado que este tipo de sistema TOA basado en un solo detector es muy útil para estudios de prueba de concepto debido a su simplicidad, bajo costo y efectividad [5], [15]. Las señales interferométricas (SI) generadas por el bloque OS son detectadas por el bloque O/E que consta de dos fotodetectores balanceados y un amplificador de transimpedancia. La señal eléctrica de este bloque es enviada al bloque SDH que realiza la demodulación de la señales interferométricas. Finalmente, los datos son enviados a la PC que se encarga de realizar la reconstrucción de la imagen. Una descripción más detallada de la configuración experimental se encuentra en [13].

\section{B. Detector de ultrasonido}

Como se mencionó más arriba, la detección de las señales OA se realiza con un sensor óptico basado en un interferómetro Mach-Zehnder en espacio libre que se comporta como un detector ultrasónico lineal extenso (haz del láser $\mathrm{HeNe}$ dentro del volumen de control). La onda de presión generada por efecto OA, $p(\mathbf{r}, t)$, produce una variación del índice de refracción dada por la siguiente expresión [13],

$$
\Delta n(\mathbf{r}, t)=\frac{d n}{d p} p(\mathbf{r}, t)
$$

donde para agua destilada $d n / d p=1.35 \cdot 10^{-10} \mathrm{~Pa}^{-1}$ [6]. Para obtener el cambio de fase medido, se integra la presión a lo largo del detector óptico lineal de largo $L$ [6]:

$$
\Delta \phi(t)=\frac{2 \pi}{\lambda} \frac{d n}{d p} \int_{L} p(\mathbf{r}, t) d L
$$

Sin embargo, un sensor lineal real posee un volumen de detección finito. En particular, para el arreglo interferométrico heterodino estudiado en este trabajo, el sensor es el haz láser (HeNe) que posee un perfil de intensidad gaussiano $I(\mathbf{r})$ correspondiente a la rama de detección que atraviesa el recipiente que contiene la muestra. De esta manera, la variación de fase en función de tiempo para un detector real es [6]:

$$
\Delta \phi(t)=\frac{2 \pi}{\lambda U_{0}} \frac{d n}{d p} \int_{V} p(\mathbf{r}, t) I(\mathbf{r}) d V
$$

donde $U_{0}$ es la potencia del haz láser.

Con el objetivo de analizar el efecto que tiene el uso de un detector de volumen finito sobre la señal OA, se llevó a cabo la simulación que se detalla a continuación. Para comparar las respuestas obtenidas de los sensores lineales ideal y real, se simuló la onda acústica emitida por una fuente esférica lo suficientemente pequeña para poder considerarla puntual (en este caso $<100 \mu \mathrm{m}$ ) ubicada a la distancia de $12 \mathrm{~mm}$ del detector. La presión acústica generada por una fuente esférica de radio $a$ iluminada de manera uniforme y ubicada en el origen de coordenadas está dada por la siguiente expresión [16]:

$$
p(\mathbf{r}, t)=2 p_{0} H\left(a-\left|r-v_{s} t\right|\right) \frac{r-v_{s} t}{2 r}
$$

donde $p_{0}$ es la presión acústica inicial, $v_{s}$ la velocidad del sonido en el medio líquido y $H$ es la función escalón de Heaviside. El detector óptico se modeló como un cilindro de $1 \mathrm{~mm}$ de diámetro y $100 \mathrm{~mm}$ de longitud total. En la Fig. 2 se presenta la respuesta simulada de un sensor lineal con y sin volumen despreciable (ideal y real, respectivamente).

Conceptualmente, el sensor interferométrico lineal real, a diferencia del ideal que se puede modelar como una línea recta de longitud finita, sumará en el interior de su volumen los ciclos positivos de presión con los negativos, cancelándolos unos con otros. Por lo tanto, es de esperar que a frecuencias acústicas suficientemente altas, tales que su longitud de onda sea menor que el diámetro del haz de sensado, se produzca un efecto similar al de un filtro pasabajos (ver Fig. 2). Dado que el haz láser posee un perfil gaussiano, es razonable intentar modelar este efecto como un filtro pasabajos gaussiano con una frecuencia de corte del orden del tiempo que le toma a la onda acústica transitar la sección transversal del haz láser. Otra variable de ajuste que se tuvo en cuenta fue el parámetro de truncamiento del filtro, donde el valor que mejor ajusta es 1.5 veces la desviación estándar. El filtro se implementó usando el modulo de Python gaussian_filterld. En la Fig. 2 se puede observar una buena concordancia entre la señal simulada considerando un volumen finito y la obtenida luego de aplicar un filtro gaussiano truncado a la señal devuelta por el sensor lineal ideal. De esta manera se tiene una forma sencilla de modelar este efecto en la generación de señales OA para entrenamiento de redes neuronales.

\section{Ruido eléctrico}

Para caracterizar el ruido eléctrico presente en el sistema TOA se adquirieron señales usando una muestra que no absorbe la radiación incidente y que, por lo tanto, no genera una presión acústica. En las Figs. 3 y 4 se muestra un ejemplo del ruido eléctrico medido en función del tiempo y la frecuencia, respectivamente. En particular, en el espectro de las señales de ruido adquiridas se observan componentes sub-armónicas de la frecuencia de 75 MHZ usada en el proceso de heterodinaje. Por ejemplo, la componente de $25 \mathrm{MHZ}$, correspondiente a un tercio de la frecuencia de modulación, está presente en todos los espectros de potencia observados, tal como se puede apreciar en la Fig. 4. 


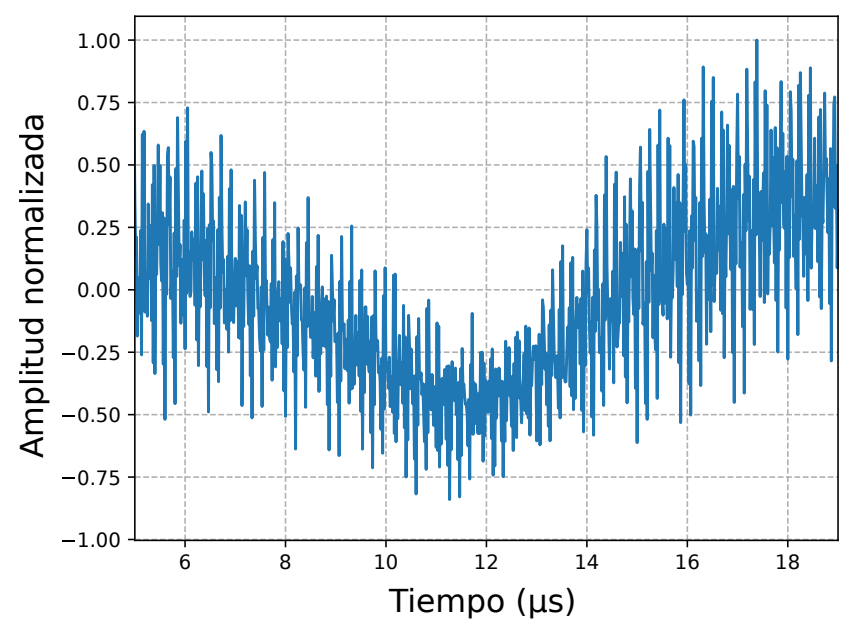

Fig. 3. Ejemplo de una medición de ruido.

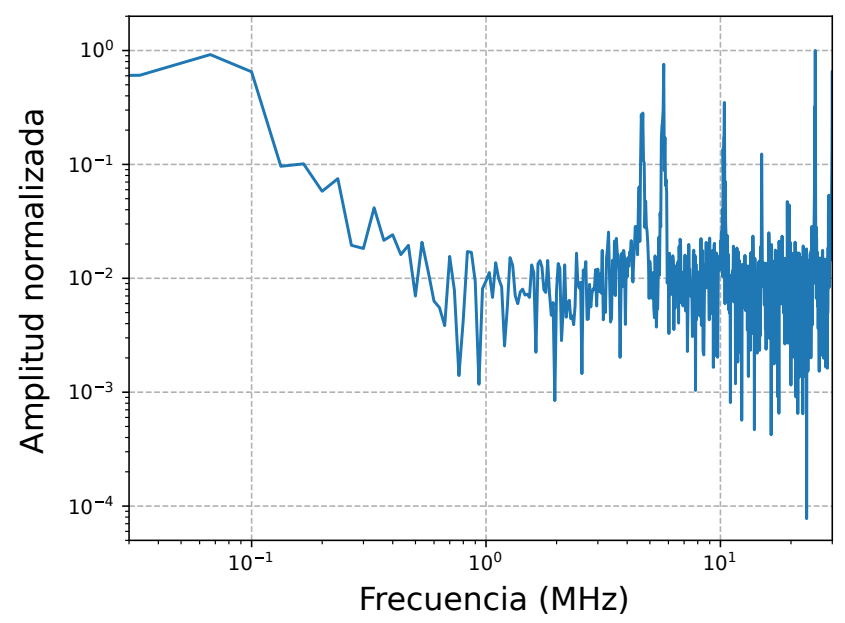

Fig. 4. Espectro normalizado de la señal de ruido presentada en la Fig. 3

Para generar señales OA que contengan el ruido eléctrico del sistema bajo estudio, se optó por el siguiente esquema de simulación que consta de 4 pasos: (i) determinar las amplitudes máximas y mínimas de cada una de las señales de ruido medidas (64 señales); (ii) elegir una de las señales medidas al azar; (iii) normalizar; (iv) asignar un valor de amplitud aleatorio en el rango entre el máximo y mínimo medidos. De esta forma, queda garantizado que el espectro de frecuencias de las señales permanecerá inalterado.

\section{Modelo matricial}

En este trabajo se utilizó el enfoque matricial (MM) tanto para la reconstrucción de las imágenes (problema inverso) como para la simulación de la señales de presión detectadas por el sensor lineal en distintas ubicaciones alrededor de la muestra (problema directo). El método MM está basado en la representación discreta del modelo acústico directo que describe la propagación del pulso de presión generado por el efecto OA. Este permite la construcción de una matriz, que representa el problema directo y las características de la configuración del sistema para TOA. Con este enfoque, la reconstrucción de la imagen se realiza minimizando numéricamente el error (normalmente cuantificado mediante una función de pérdida cuadrática) entre las señales acústicas medidas y las predichas utilizando el modelo acústico directo [17]. Aunque los algoritmos basados en MM tienden a ser computacionalmente intensivos, ya que requieren calcular y manipular matrices muy grandes [18], éstos son más versátiles dado que se pueden aplicar a geometrías de medición arbitrarias y se pueden agregar muchos efectos lineales adicionales al modelo [19]. Esta última característica es la razón principal de haber elegido este enfoque.

En el dominio del tiempo, el sistema matricial del problema directo es [17]:

$$
\mathbf{p}_{\mathbf{d}}=\mathbf{A} \mathbf{p}_{\mathbf{0}}
$$

donde $\mathbf{p}_{\mathbf{d}} \in \mathbb{R}^{N_{d} \cdot N_{t} \times 1}$ es un vector que representa las presiones medidas en un conjunto de ubicaciones $\mathbf{r}_{\mathbf{d} l}(l=$ $\left.1 \ldots N_{d}\right)$ en los instantes de tiempo $t_{k}\left(k=1 \ldots N_{t}\right)$; $\mathbf{p}_{\mathbf{0}} \in \mathbb{R}^{N \times 1}$ es un vector que representa los valores de la presión acústica inicial en la cuadrícula de la región de imagen; y $\mathbf{A} \in \mathbb{R}^{N_{d} \cdot N_{t} \times N}$ es la matriz del modelo. El $j$-ésimo elemento $(j=1 \ldots N)$ en $\mathbf{p}_{0}$ contiene el valor promedio de la presión inicial dentro de un elemento de superficie en la posición $\mathbf{r}_{j}$ de la región imagen. Una vez que se ha establecido la formulación discreta, el problema inverso se reduce al problema algebraico de invertir el sistema lineal. Una descripción detallada del algoritmo utilizado en este trabajo se encuentra en [20].

Para definir los valores de los parámetros para las simulaciones, se tuvo en cuenta la configuración experimental utilizada en las mediciones realizadas con el sistema TOASDO descripto en la subsección II-A. Se definió una región imagen cuadrada con un tamaño de $8 \times 8 \mathrm{~mm}^{2}$ y una resolución de 128 x 128 píxeles $(N=16384)$. El fantoma usado es una imagen 2-D (letras capitales OB) cuya imagen se presenta en la Fig. 5.a. Tanto la muestra como el detector se encuentra sumergidos en agua destilada. La velocidad del sonido en el agua se fijó en $v_{s}=1485 \mathrm{~m} / \mathrm{s}$ y este medio acústico se supuso homogéneo y sin absorción o dispersión del sonido. Las señales OA se detectaron en $N_{d}=120$ ubicaciones sobre una circunferencia de $\sim 12 \mathrm{~mm}$ de radio cuyo centro se encuentra en el eje de la plataforma giratoria donde se encuentra dispuesto el fantoma. El detector lineal tiene una largo de $100 \mathrm{~mm}$ y en la matriz del modelo se supuso que su volumen es despreciable. Para la recopilación de datos, el intervalo de tiempo $\Delta t$ fue de $15 \mathrm{~ns}$ con $N_{t}=800$ muestras. Las simulaciones se llevaron a cabo en Python. Para obtener las imágenes a partir de las presiones $\mathbf{p}_{\mathbf{d}}$ se emplea el algoritmo $l s q r$ proporcionado en el módulo de Python scipy.sparse.linalg que utiliza un método iterativo para aproximar la solución del ec. 5. Como figura de mérito para comparar las señales obtenidas, se empleó el índice de similitud estructural (SSIM por sus siglas en inglés) que combina tres medidas comparativas (luminancia, contraste y estructura) para evaluar la calidad de la imagen obtenida con respecto a una referencia (Fig. 5.a) [21]

\section{RESUlTADOS}

En la Fig. 5.b se muestra la imagen reconstruida usando el enfoque MM a partir de la señales medidas por el sistema bajo estudio. Cualitativamente se puede observar un efecto de suavizado de los bordes y una pérdida notoria del 
(a)

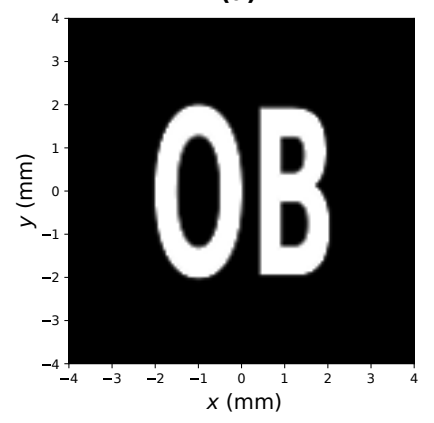

(c)

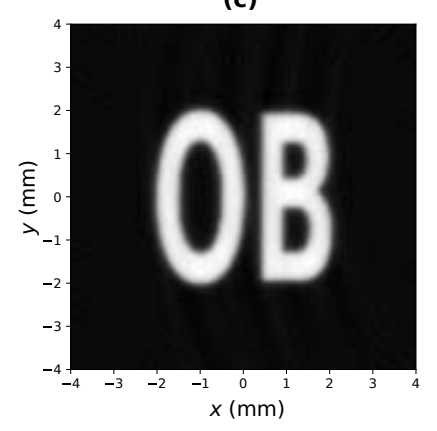

(e)

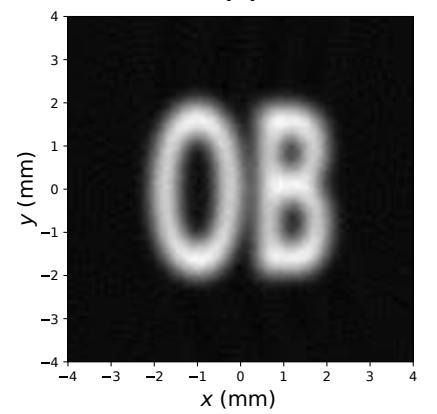

(b)

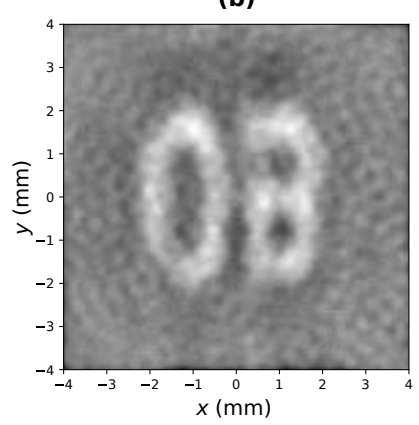

(d)

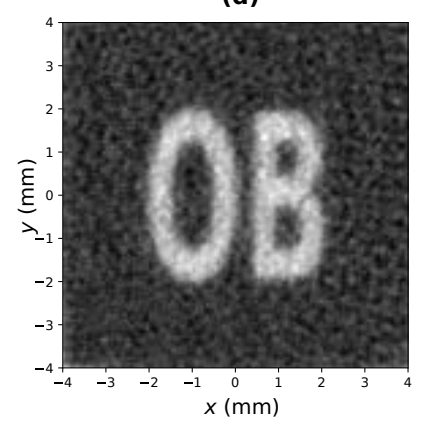

(f)

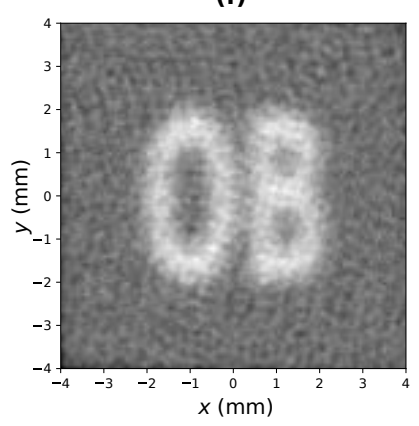

Fig. 5. (a) Imagen original. (b) Imagen obtenida a partir de las presiones medidas. (c)-(f) Reconstrucciones obtenidas a partir de simulaciones para diferentes condiciones. (c) Sensor lineal ideal y sin ruido. (d) Con ruido eléctrico. (e) Sensor lineal real. (f) Sensor lineal real y con ruido.

contraste (baja SNR). Estas apreciaciones concuerden con el bajo valor de $\mathrm{SSIM}=0.135$.

En las Figs. 5.c-f se presentan las reconstrucciones obtenidas a partir de simulaciones para diferentes condiciones y utilizando los modelos de sensor real detallados en la sección II. La Fig. 5.c es la reconstrucción para el caso en que el sensor lineal es ideal y el ruido eléctrico es despreciable. La falta de contraste en la imagen es debido a la cantidad de ubicaciones $N_{d}$ usadas en la reconstrucción que hace que el valor de SSIM $=0.862$ sea elevado pero menor que el máximo esperable. En la Fig. 5.d se presenta el caso de cuando las señales simuladas son corrompidas con ruido eléctrico $(\mathrm{SNR}=18 \mathrm{~dB})$, obteniéndose una $\mathrm{SSIM}=0.265$. Por otro lado, en la Fig. 5.e se encuentra la imagen obtenida luego de aplicar el filtro gaussiano determinado en la subsección II-B donde se puede observar el efecto de suavizado producido por el volumen finito del detector óptico. En este caso, el valor de SSIM = 0.742 no se ve reducido tan drásticamente como en el caso del ruido eléctrico. Por último, en la Fig. 5.f se presenta el efecto combinado, donde primero se filtran las señales simuladas

con el modelo matricial y luego se agrega el ruido eléctrico. De esta manera, se llega a una imagen cualitativamente y cuantitativamente $($ SSIM $=0.194)$ muy parecida a la reconstruida a partir de las mediciones.

\section{CONCLusiones}

En este trabajo se analizaron y caracterizaron las causas de los artefactos introducidos en las imágenes obtenidas con un sistema para TOA desarrollado previamente [13] y que se encuentra basado en el concepto de SDO y en la detección de ultrasonido usando interferometría óptica heterodina. La baja calidad en las imágenes obtenidas se debe principalmente a la forma del sensor ultrasónico y a la presencia de ruido eléctrico. Por un lado, el haz láser, utilizado para detectar los cambios de índice de refracción generados por la muestra, tiene un volumen no despreciable que causa un efecto de suavizado de bordes en las imágenes obtenidas. Luego de realizar un conjunto de simulaciones, se encontró que este efecto puede ser modelado mediante un filtro gaussiano truncado con una frecuencia de corte en concordancia con el tiempo de tránsito de la onda acústica por la sección transversal del haz. Por otro lado, el ruido eléctrico es debido a la electrónica de la SDH. Para simular este problema, se utilizaron señales de ruido medidas para construir versiones simuladas con el mismo contenido espectral de frecuencia. Para probar el rendimiento de estos modelos que tienen en cuenta la forma del detector y el ruido eléctrico, se comparó cualitativa y cuantitativamente las imágenes reconstruidas a partir de simulaciones con aquella imagen obtenida de las señales $\mathrm{OA}$ medidas con el sistema bajo estudio. Como objetivo a futuro, se espera utilizar estos modelos para generar señales simuladas que sirvan para entrenar redes neuronales profundas que mejoren la calidad de las imágenes reconstruidas [2].

\section{AGRADECIMIENTOS}

Este trabajo fue apoyado por los subsidios de la ANPCyT (PICT 2018-04589), del CONICET (PIP $11220200101826 \mathrm{CO}$ ) y de la Universidad de Buenos Aires (UBACYT 20020190100032BA).

\section{REFERENCIAS}

[1] L. V. Wang and H. Wu, Biomedical Optics: Principles and Imaging. John Wiley \& Sons, 2009.

[2] A. Hauptmann and B. Cox, "Deep learning in photoacoustic tomography: current approaches and future directions," Journal of Biomedical Optics, vol. 25, no. 11, pp. $1-46,2020$.

[3] M. G. Gonzalez, E. Acosta, and G. Santiago, "Simple method to determine the resolution and sensitivity of systems for optoacoustic tomography," Elektron, vol. 2, pp. 63-66, 2018.

[4] A. F. Vidal, L. C. Brazzano, C. Matteo, P. Sorichetti, and M. G. Gonzalez, "Parametric modeling of wideband piezoelectric polymer sensors: design for optoacoustic applications," Rev. Sci. Instrum., vol. 88, no. 9, p. 095004, 2017.

[5] C. Tian, M. Pei, K. Shen, S. Liu, Z. Hu, and T. Feng, "Impact of system factors on the performance of photoacoustic tomography scanners," Phys. Rev. Applied, vol. 13, p. 014001, 2020.

[6] G. Paltauf, R. Nuster, and P. Burgholzer, "Characterization of integrating ultrasound detectors for photoacoustic tomography," Journal of Applied Physics, vol. 105, 2009.

[7] M. G. Gonzalez, L. Riobo, L. C. Brazzano, F. Veiras, P. Sorichetti, and G. Santiago, "Generation of sub-microsecond quasi-unipolar pressure pulses," Ultrasonics, vol. 98, pp. 15-19, 2019.

[8] S. Tzoumas, A. Rosenthal, C. Lutzweiler, D. Razansky, and V. Ntziachristos, "Spatiospectral denoising framework for multispectral optoacoustic imaging based on sparse signal representation," Medical Physics, vol. 41, p. 113301, 2014. 
[9] J. Barry, E. Lee, and D. Messerschmitt, Digital communication. Springer Science and Business Media, 2012.

[10] C. Dehner, I. Olefir, K. Chowdhury, D. Juestel, and V. Ntziachristos, "Deep learning based electrical noise removal enables high spectral optoacoustic contrast in deep tissue," arXiv, 2021.

[11] B. Cox, J. Laufer, S. Arridge, and P. Beard, "Quantitative spectroscopic photoacoustic imaging: a review," Journal of Biomedical Optics, vol. 17, p. 061202, 2012.

[12] L. Zeng, D. Xing, H. Gu, D. Yang, S. Yang, and L. Xiang, "High antinoise photoacoustic tomography based on a modified filtered backprojection algorithm with combination wavelet," Medical Physics, vol. 34, pp. 556-563, 2007.

[13] R. Insabella, M. Gonzalez, R. Riobo, K. Hass, and F. Veiras, "Software-defined optoacoustic tomography," Appl. Opt., vol. 59, pp. 706-711, 2020.

[14] L. Riobo, F. Veiras, M. G. Gonzalez, M. T. Garea, and P. Sorichetti, "High-speed real-time heterodyne interferometry using softwaredefined radio," Appl. Opt., vol. 57, no. 2, pp. 217-224, 2017.

[15] A. Sharma, S. Kalva, and M. Pramanik, "A comparative study of continuous versus stop-and-go scanning in circular scanning photoacoustic tomography," IEEE J. Sel. Top. Quantum Electron, vol. 25, no. 1, pp. 1-9, 2019.
[16] M. Xu, Y. Xu, and L. Wang, "Time-domain reconstruction algorithms and numerical simulations for thermoacoustic tomography in various geometries," IEEE Transactions on Biomedical Engineering, vol. 50, pp. 1086-1099, 2003.

[17] A. Rosenthal, V. Ntziachristos, and D. Razansky, "Acoustic inversion in optoacoustic tomography: A review," Current Medical Imaging Reviews, vol. 9, pp. 318-336, 2013.

[18] L. Ding, X. Dean-Ben, and D. Razansky, "Real-time model-based inversion in cross-sectional optoacoustic tomography," IEEE Trans Med Imaging, vol. 35, pp. 1883-1891, 2016.

[19] A. Rosenthal, D. Razansky, and V. Ntziachristos, "Fast semi-analytical model-based acoustic inversion for quantitative optoacoustic tomography," IEEE Trans Med Imaging, vol. 29, no. 6, pp. 1275-1285, 2010.

[20] L. Hirsch, M. G. Gonzalez, and L. R. Vega, "On the robustness of model-based algorithms for photoacoustic tomography: comparison between time and frequency domains," Rev. Sci. Instrum., vol. 92, p. 114901, 2021.

[21] Z. Wang, A. Bovik, H. Sheikh, and E. Simoncelli, "Image quality assessment: from error visibility to structural similarity," IEEE Transactions on Image Processing, vol. 13, no. 4, p. 600-612, Apr 2004. 